\title{
Three-Dimensional Magnetic Resonance Arthrography of Post-Arthroscopy Hip Instability Demonstrates Increased Effective Intracapsular Volume and Anterosuperior Capsular Changes
}

\author{
Joseph Featherall, M.D., Dillon C. O'Neill, M.D., Alexander J. Mortensen, M.D., \\ Kelly M. Tomasevich, B.A., Allan K. Metz, B.S., and Stephen K. Aoki, M.D.
}

\begin{abstract}
Purpose: To quantify the magnetic resonance arthrography (MRA) capsular morphologic findings associated with postarthroscopy hip instability. Methods: Among patients with clinically significant iatrogenic hip instability at a single center, patients with preindex and postindex surgery MRAs were identified. These MRAs were compared regarding effective intracapsular volume calculated by semi-automated 3-dimensional pixel intensity region segmentation, 2dimensional anterior proximal intracapsular area in the femoral neck axial plane reconstruction, maximal anterior fluid pocket depth, capsule retraction distance, and capsular instability grade. Morphological measurements were conducted using Horos image processing software. Paired $t$-test, paired Wilcoxon signed rank test, and the McNemar test were used for identifying statistical significance. Results: In 42 patients, mean effective intracapsular volume was significantly greater in the postindex surgery MRAs $\left(19.44 \mathrm{~cm}^{3}\right.$ vs $\left.17.26 \mathrm{~cm}^{3} ; P=.006\right)$. Proximal anterosuperior $(12-3$ o'clock) intracapsular area was also significantly greater after index surgery $\left(2.84 \mathrm{~cm}^{2}\right.$ vs $1.43 \mathrm{~cm}^{2} ; P<.001$. Proximal anteroinferior (3-6 o'clock) intracapsular area $\left(1.34 \mathrm{~cm}^{2}\right.$ vs $\left.0.97 \mathrm{~cm}^{2} ; P=.002\right)$, capsule deficiency grade $(P<.001)$, anterior capsule retraction distance $(4.83 \mathrm{~mm}$ vs $0.34 \mathrm{~mm} ; P<.001)$, and maximum anterior fluid depth $(8.33 \mathrm{~mm}$ vs $4.90 \mathrm{~mm} ; P$ $<.001)$ were also significantly increased after index surgery. Conclusion: In comparison to the preoperative state, iatrogenic hip instability is associated with MRA findings that include increases in total effective intracapsular volume, proximal anterosuperior and anteroinferior intracapsular cross-sectional area, maximum proximal anterosuperior fluid depth, and capsule retraction distance. Level of Evidence: Level IV, diagnostic case series.
\end{abstract}

$\mathbf{W}$ ith increasing prevalence of hip arthroscopy in the United States and globally, there is increasing recognition of the complications of these procedures

From the University of Utah, Department of Orthopaedics, Salt Lake City, Utah, U.S.A.

The authors report the following potential conflicts of interest or sources of funding: D.C.O. reports personal fees from Active Medical LLC and Acumed LLC. S.K.A. reports personal fees from Stryker Corporation, Active Medical LLC, and Smith $\theta$ Nephew Inc. Full ICMJE author disclosure forms are available for this article online, as supplementary material.

The data from this article has been previously presented at Orthopaedic Summit Emerging Technologies in December 2020.

Received February 2, 2021; accepted September 28, 2021.

Address correspondence to Stephen K. Aoki, M.D., Department of Orthopaedic Surgery, University of Utah, 590 Wakara Way, Salt Lake City, UT 84108.E-mail: stephen.aoki@hsc.utah.edu

(C) 2021 THE AUTHORS. Published by Elsevier Inc. on behalf of the Arthroscopy Association of North America. This is an open access article under the CC BY-NC-ND license (http://creativecommons.org/licenses/by-nc-nd/4.0/).

2666-061X/21163

https://doi.org/10.1016/j.asmr.2021.09.022 and the need for revision surgery in some cases. ${ }^{1} \mathrm{In}$ dications for secondary surgeries are well known, including end-stage arthritis, under-resection of cam deformities, failure of labral repair/healing, and hip dislocation. $^{2-5}$ Although previous studies have shown high rates of capsular healing with and without capsular repair, it has also been shown that a certain portion of patients will have capsular deficiencies after arthroscopy. ${ }^{6-9}$ Hip instability, because of inadequate capsular closure/healing or reinjury, is an increasingly prevalent indication for revision hip arthroscopy, although it is currently less understood than other revision indications. ${ }^{10-13}$

As the recognition of hip instability increases, there is a growing appreciation for its treatment options, which include simple capsular repair, capsular plication, or capsular reconstruction. ${ }^{14,15}$ There is limited understanding of the ideal methods for diagnosis of postarthroscopy hip instability. ${ }^{12,16,17}$ Furthermore, the mechanisms of pain generation and the effects on 

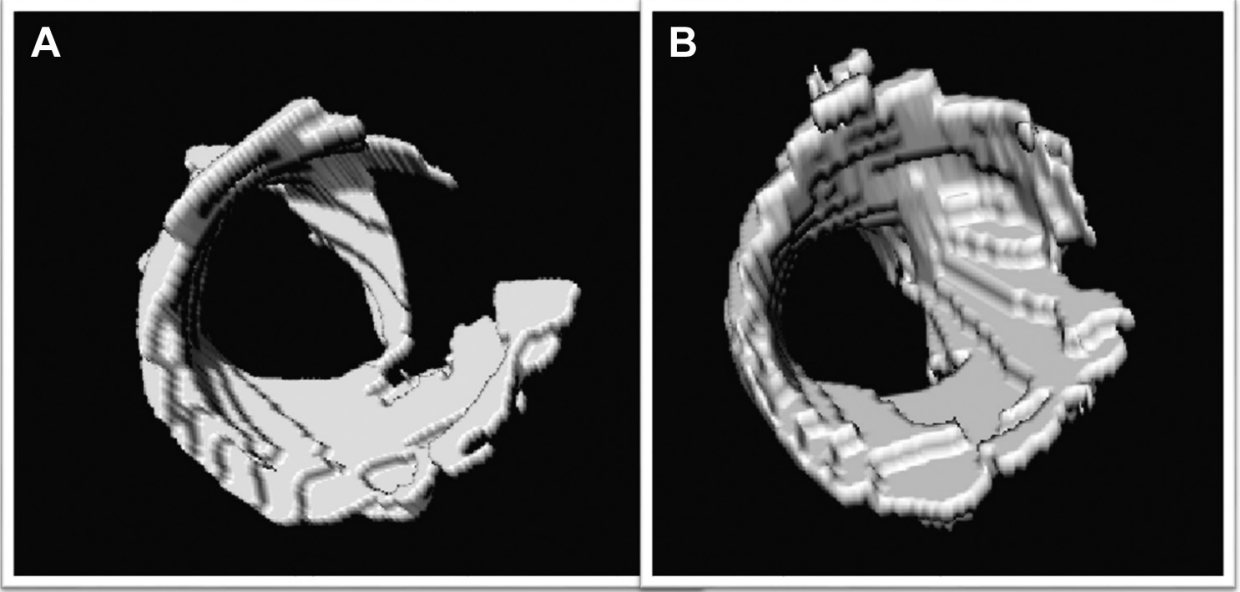

Fig 1. Left hip effective intracapsular volume renderings from a single patient viewing medialto-lateral. (A) Preindex surgery volume demonstrates no contrast in the region of a native iliofemoral ligament. (B) Postindex surgery volume rendering demonstrates increased patulousness/ contrast volume in the iliofemoral ligament region.

biomechanics in the long-term health of the hip joint are also not fully understood.

Magnetic resonance imaging (MRI), including magnetic resonance arthrography (MRA), is a key imaging modality for diagnosing and assessing nonarthritic hip pathology. ${ }^{9,12}$ There have been a number of studies that have used MRI to identify and characterize capsular healing after hip arthroscopy for femoroacetabular impingement. ${ }^{8,9}$ These studies offer key insights into the natural history of capsular healing; however, the pathologic or unstable hip capsular morphology has not yet been closely studied with MRA. Thus far, only small case series and qualitative discussions of magnetic resonance assessments of unstable capsules have been described. ${ }^{7,12,18}$

MRA offers distinct advantages over MRI and other imaging modalities for identifying hip capsular pathology. Intra-articular contrast injection distends the capsule allowing the radiologist or orthopaedic surgeon to assess for patulous capsular morphology and overall capsular volume. The intra-articular contrast also further highlights gross extravasation, redundancy, and focal rents within the capsule, and clearly defines overall capsular morphology. ${ }^{18}$

The purpose of this study was to rigorously quantify the MRA capsular morphologic findings associated with post-arthroscopy hip instability. The authors hypothesized that postarthroscopy hip instability would be associated with a number of MRA findings, including increased capsular volume, maximum anterior fluid pocket depth, capsular retraction distance, proximal intracapsular area in the femoral neck axial plane, and capsular deficiency grade.

\section{Methods}

\section{Patient Selection}

All patients who underwent revision hip arthroscopy for iatrogenic hip instability at a single institution by the senior author (S.K.A.) between February 2011 and December 2019 were identified and reviewed. Inclusion criteria were revision hip arthroscopy with iatrogenic instability as an indication and available before index surgery MRA, and available after index surgery, before

Fig 2. Axial MRAs of superior head neck junction of the left hip of the same patient. (A) Preindex surgery showing tensioned iliofemoral ligament with minimal anterior volume. (B) Postindex surgery MRA showing increased anterosuperior segmentation area.
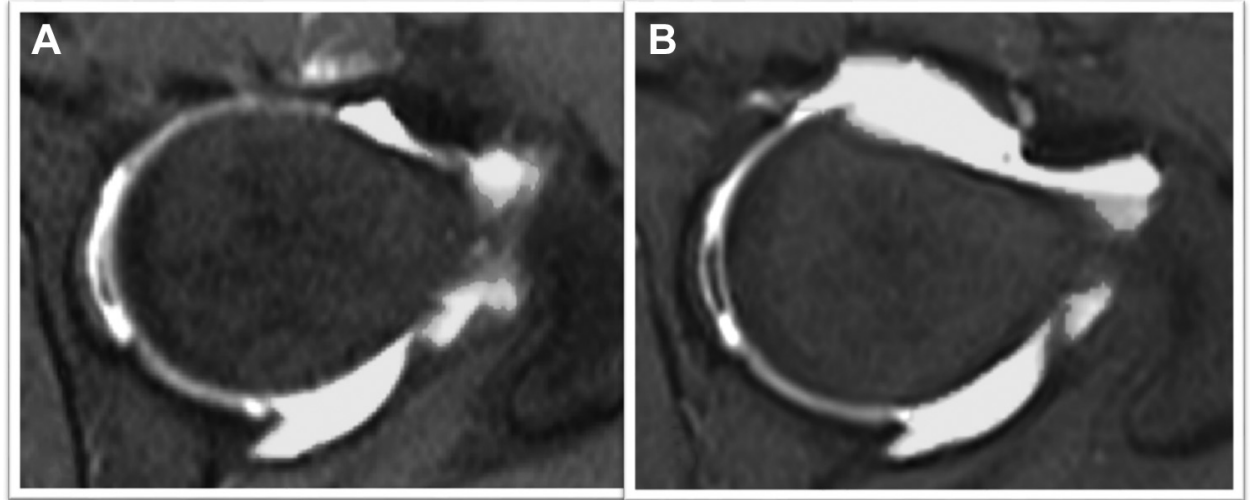

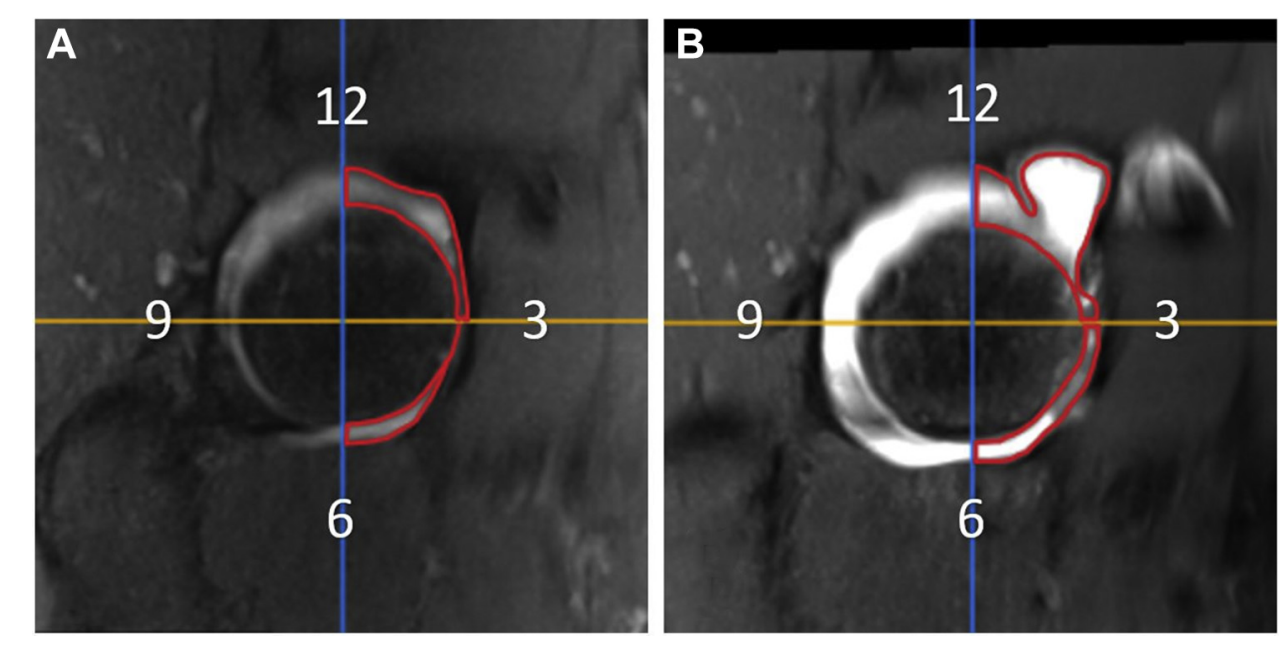

Fig 3. Gunsight reconstructions of right hip MRAs at the femoral head-neck junction of the same patient before and after index hip arthroscopy surgery. Intracapsular area within the 12-3 and 3-6 o'clock regions are shown outlined in red. (A) Preindex surgery. (B) Postindex surgery.

revision surgery MRA. Exclusion criteria were patients undergoing revision hip arthroscopy for other indications and patients without available MRA imaging. Indications for surgery and clinical criteria for postarthroscopy hip instability were as previously published. ${ }^{18}$ Institutional review board approval was obtained for this study.

\section{MRA Data Collection and Validation}

Preindex and postindex arthroscopy MRAs were compiled into a database. This database was then accessed using Horos image processing software (horosproject.org, Purview, Annapolis, MD). MRA sequences used for evaluation included sagittal proton density fat saturated and axial Tl fat-saturated sequences. A growing region volume segmentation algorithm was used to calculate effective intracapsular volume in each of the hips (Fig 1 and Fig 2). This method has been previously validated for 3-dimensional volume calculation in multiple anatomic regions. ${ }^{19-21}$ Through multiple iterations and consensus, the authors developed the following algorithm for the volume measurement: (1) cotyloid fossa volume and contrast volume inferomedial to the transverse acetabular ligament are excluded; (2) extravasation through a capsule defect contained by fascia or pseudocapsule was included; (3) infiltration into surrounding tissue without capsular defect was not included in the volume; and (4) extravasation into the psoas sheath was included only when it communicated with a capsule defect clearly identifiable on multiple MRA sequences and planes. Three-dimensional multiplane reconstructions were rendered from the sagittal MRA data to assess the intracapsular area along the proximal femoral neck in both the anterosuperior (12-3 o'clock) and the anteroinferior (3-6 o'clock) positions (Fig 3). Manual region of interest identification was conducted for calculation of these areas. Capsular insufficiency grading was assessed by the reviewer as previously described. ${ }^{18}$ Capsule retraction distance was calculated as the distance between the acetabular leaflet and the femoral leaflet
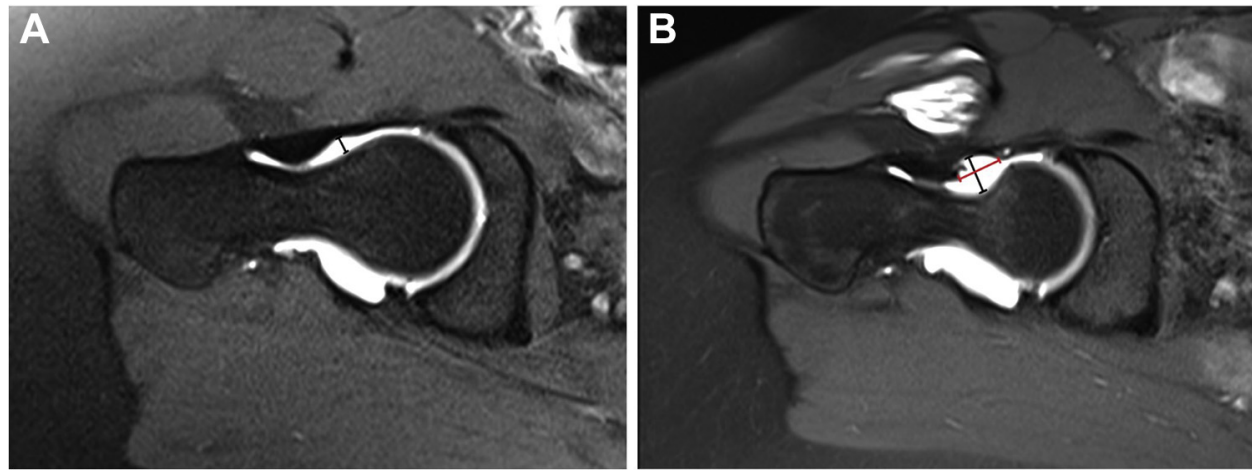

Fig 4. Axial oblique MRAs (axial plane adjusted to be in plane with femoral neck axis) of the right hip of the same patient before and after hip arthroscopy surgery. (A) Preindex surgery with maximum anterior fluid depth (black line) measured in the anterosuperior region of the femoral neck. Native hip capsule is intact; therefore no capsular retraction is present. (B) Postindex surgery with increased maximum anterior fluid depth (black line) in the anterosuperior region of the femoral neck. Capsular retraction (red line) was measured as the distance between acetabular and femoral capsular leaflets. 
when a gross capsular defect was identified in either the axial or oblique axial views (axial plane adjusted to be in plane with femoral neck axis) (Fig 4). Maximum anterior fluid depth was calculated as the maximal fluid pocket depth in the anterosuperior region of the neck on the axial or oblique axial MRAs (Fig 4).

Image analysis methods were designed and revised in an iterative consensus process among all authors. All measurements were conducted by the authors D.C.O., A.J.M., and J.F., who were trained in standardization of the image measurements. Ten cases (20 MRAs) were randomly selected and measured by all 3 reviewers for assessment of inter-rater reliability.

\section{Statistical Analysis}

All statistical analyses were conducted in R statistical software (R Foundation for Statistical Computing, Vienna, Austria). For continuous variables, the paired $t$ test was used for comparison of preindex surgery and postindex surgery capsular measurements. For categorical variables, the paired-sample Wilcoxon test was used. For binary variables, the McNemar test was used. Alpha level of 0.05 was used to determine statistical significance. Intraclass correlation coefficient (ICC) was calculated to assess reliability across the 3 reviewers. An ICC value greater than 0.75 was considered excellent, 0.60-0.74 was considered good, 0.40-0.59 was considered fair, and less than 0.40 was considered poor. ${ }^{22}$

\section{Results}

\section{Demographics and Treatment Characteristics}

Two hundred ninety-three patients underwent revision hip arthroscopy between March 2011 and December 2019. Forty-two patients were identified to meet inclusion criteria, for a total of 84 MRAs included for analysis. Patient demographics and surgical characteristics are presented in Table 1. Mean age at revision surgery was 30.5 years, and $88.1 \%$ of the cohort was female. Mean BMI was 25.0. Mean Beighton score was 1.8 , with $72.4 \%$ of patients having a Beighton score of $<4$. The vast majority of patients underwent interportal capsulotomy $(\mathrm{n}=39,95.1 \%)$, and underwent capsular closure at index procedure $(\mathrm{n}=36,85.4 \%)$. All patients underwent either capsular repair $(\mathrm{n}=33,78.6 \%)$ or capsular reconstruction $(\mathrm{n}=9,21.4 \%)$ at the time of revision surgery. Frequent, concomitantly performed procedures included lysis of adhesions $(\mathrm{n}=24,57.1 \%)$ femoral osteochondroplasty $(\mathrm{n}=10,23.8 \%)$, loose body removal $(\mathrm{n}=2,4.8 \%)$, and labral repair $(\mathrm{n}=2,4.8 \%)$.

\section{Capsule Morphology Before and After Index Surgery}

Several capsular morphological changes were identified between the preindex and postindex surgery cohorts (Table 2). Effective intracapsular volume was significantly greater in the postindex surgery group
Table 1. Demographics and Treatment Characteristics

\begin{tabular}{lc}
\hline \multicolumn{1}{c}{ Variable } & $\mathrm{n}=42$ \\
\hline Age at revision surgery, mean (SD) & $30.5(11.7)$ \\
BMI at revision surgery, mean (SD) & $25.0(4.2)$ \\
Sex & \\
Female & $37(88.1 \%)$ \\
Male & $5(11.9 \%)$ \\
Beighton score, mean (SD) & $1.8(2.9)$ \\
$<4$ & $21(72.4 \%)$ \\
$\geq 4$ & $8(27.6 \%)$ \\
Primary surgery capsulotomy & \\
T-type & $2(4.9 \%)$ \\
Interportal & $39(95.1 \%)$ \\
Primary surgery capsular repair & \\
None & $6(14.6 \%)$ \\
Repair & $33(80.5 \%)$ \\
Plication & $2(4.9 \%)$ \\
Primary surgery number of sutures used, mean (SD) & $3.2(1.9)$ \\
Primary surgery capsular repair technique & \\
Simple interrupted & $25(73.5 \%)$ \\
Figure-of-eight & $6(17.6 \%)$ \\
Other & $3(8.8 \%)$ \\
Revision surgical treatment & \\
Capsule repair & $33(78.6 \%)$ \\
Capsule reconstruction & $9(21.4 \%)$ \\
Femoral osteochondroplasty & $10(23.8 \%)$ \\
Acetabuloplasty & $1(2.4 \%)$ \\
Labral repair & $2(4.8 \%)$ \\
Labral reconstruction & $1(2.4 \%)$ \\
Lysis of adhesions & $24(57.15)$ \\
Loose body removal & $2(4.85)$ \\
Psoas release & $1(2.4 \%)$ \\
\hline BMI, body mass index & \\
All variables reported as mean (SD) unless otherwise & specified. \\
&
\end{tabular}

$\left(19.54 \pm 5.51\right.$ vs $\left.17.26 \pm 5.23 \mathrm{~cm}^{3} ; \mathrm{P}=.006\right)$. Proximal anterosuperior intracapsular area (12-3 o'clock area) was significantly greater at the postindex surgery time point $\left(2.84 \pm 0.82\right.$ vs $\left.1.43 \pm 0.75 \mathrm{~cm}^{2} ; \mathrm{P}<.001\right)$, as was the proximal anteroinferior intracapsular area (3-6 $\mathrm{o}^{\prime}$ clock area) $(1.34 \pm 0.59$ vs $0.97 \pm 0.49 ; \mathrm{P}=.002)$. Capsule insufficiency grade was significantly greater at the post index surgery time point, as was capsule retraction distance $(4.71 \pm 6.53$ vs $0.34 \pm 2.18 \mathrm{~mm} ; \mathrm{P}<$ $.001)$, and maximum anterior fluid depth $(8.33 \pm 4.44$ vs $4.90 \pm 2.67 \mathrm{~mm} ; \mathrm{P}<.001)$.

\section{Agreement}

Intraclass correlation coefficients were graded as excellent for effective intracapsular volume $(0.84 ; 95 \%$ confidence interval [CI] 0.60 to 0.94$)$, proximal anterosuperior intracapsular area $(0.86 ; 95 \%$ CI 0.64 to $0.95)$, capsule grade $(0.86 ; 95 \%$ CI 0.73 to 0.94$)$, and maximum anterior fluid depth $(0.79 ; 95 \%$ CI 0.61 to 0.90 ) as shown in Table 3. Capsule retraction distance was determined to be fair reliability $(0.40 ; 95 \%$ CI 0.13 to 0.66 ). Proximal anteroinferior intracapsular area reliability was rated as poor $(0.23 ; 95 \%$ CI -0.01 to $0.52)$. 
Table 2. Hip Capsule MRA Morphological Characteristics

\begin{tabular}{|c|c|c|c|}
\hline & Before Index Surgery & After Index Surgery & $P$ value \\
\hline $\bar{n}$ & 42 & 42 & \\
\hline Effective intracapsular volume $\left(\mathrm{cm}^{3}\right)$ & $17.26(5.23)$ & $19.54(5.51)$ & .006 \\
\hline Proximal $12-3$ o'clock capsular area $\left(\mathrm{cm}^{2}\right)$ & $1.43(0.75)$ & $2.84(0.82)$ & $<.001$ \\
\hline Capsule grade, $\mathrm{n}(\%)$ & & & $<.001$ \\
\hline 0 & $33(78.6 \%)$ & $3(7.1 \%)$ & \\
\hline 1 & $8(19.0 \%)$ & $22(52.4 \%)$ & \\
\hline Capsule retraction distance $(\mathrm{mm})$ & $0.34(2.18)$ & $4.71(6.53)$ & $<.001$ \\
\hline Maximum anterior fluid depth (mm) & $4.90(2.67)$ & $8.33(4.44)$ & $<.001$ \\
\hline
\end{tabular}

SD, standard deviation.

All variables reported as mean (SD) unless otherwise specified. Student's paired $t$ test used for continuous variable comparisons. Wilcoxon signed rank test used for ordinal variable comparisons.

\section{Clinical Examination Findings}

Prerevision and postrevision clinical examination findings and comparisons are presented in Table 4. Several clinical examination findings associated with instability improved after revision surgery with capsular reconstruction or repair. Visual analog scale (VAS) pain at rest decreased from $4.69 \pm 2.62$ to $2.91 \pm 2.67$ $(P=.004)$. Regarding pain with axial distraction, 26 patients $(70.3 \%)$ demonstrated pain before revision surgery whereas only $3(11.1 \%)$ demonstrated axial distraction pain after revision $(P<.001)$. For axial distraction apprehension testing, 13 of 42 patients $(36.1 \%)$ had apprehension before revision surgery whereas 4 of $42(14.8 \%)$ had apprehension after revision surgery. However, this difference did not reach statistical significance $(P=.228)$. Palpable toggling of the hip joint with axial distraction on examination was seen in 25 patients $(62.5 \%)$ before revision surgery and was only detectable in 3 patients $(10.7 \%)$ after revision surgery $(P=.002)$.

\section{Discussion}

This study quantifies several key capsular changes observable on MRA that are associated with clinically symptomatic postarthroscopy hip instability, including increases in effective intracapsular volume, proximal 12-3 o'clock capsular area, proximal 3-6 o'clock capsular area, capsule retraction distance, and maximum anterior fluid depth. Hip capsular morphology grading significantly differed between preindex and postindex surgery capsular states. Capsular redundancy, capsular rents, and gross extracapsular extravasation were regular findings in our postarthroscopy hip instability cohort. In addition, postoperative increases in total effective intracapsular volume, proximal anterosuperior intracapsular cross sectional area, maximal anterior fluid pocket depth, and capsular retraction distance were quantifiable MRA findings in this hip preservation patient population.

Previous research has demonstrated that MRA is a unique and powerful tool for the assessment of the hip capsule. ${ }^{23,24}$ However, hip instability after a previous hip arthroscopy can be difficult to diagnose in part because there are numerous causes for post-hip arthroscopy pain. Causes include labral/chondral pathology, inadequate cam resection, femoral or acetabular over-resection, intra-articular adhesions, soft tissue irritation of the surrounding hip musculature and tendons, and nerve pain in the general area. ${ }^{25,26}$ Currently there is no consensus regarding the clinical definition of postarthroscopy hip instability because the diagnosis is currently emerging and the field is refining its understanding of this condition. ${ }^{18,27-29}$ Clinicians rely on the patient history of subjective instability or pain with hyperextension maneuvers, physical examination

Table 3. Hip Capsule Measurement Intraclass Correlation

\begin{tabular}{lccc}
\hline \multicolumn{1}{c}{ Measurement } & Correlation Coefficient & 95\% CI Lower Bound & 95\% CI Upper Bound \\
\hline Effective intracapsular volume & 0.84 & 0.60 & 0.94 \\
Proximal 12-3 o'clock capsular area & 0.86 & 0.64 & 0.95 \\
Proximal 3-6 o'clock capsular area & 0.23 & -0.01 & 0.73 \\
Capsule grade & 0.86 & 0.13 & 0.94 \\
Capsule retraction distance & 0.40 & 0.61 & 0.66 \\
Maximum anterior fluid depth & 0.79 & 0.90 \\
\hline
\end{tabular}

\footnotetext{
CI, confidence interval.
} 
Table 4. Patient Clinical Examination Characteristics

\begin{tabular}{|c|c|c|c|}
\hline & Before Revision Surgery & After Revision Surgery & $P$ value \\
\hline $\mathrm{n}$ & 42 & 42 & \\
\hline Rest pain, VAS 0-10, mean (SD) & $4.69(2.62)$ & $2.91(2.67)$ & .004 \\
\hline Axial distraction pain & $26(70.3 \%)$ & $3(11.1 \%)$ & $<.001$ \\
\hline Axial distraction apprehension & $13(36.1 \%)$ & $4(14.8 \%)$ & .228 \\
\hline Axial distraction toggle & $25(62.5 \%)$ & $3(10.7 \%)$ & .002 \\
\hline
\end{tabular}

VAS, visual analog scale; SD, standard deviation.

Student's paired $t$ test used for continuous variable comparisons. McNemar test used for binary variable comparisons.

findings of pain, apprehension or palpable instability with distraction or hyperextension of the hip joint, as well as findings on MRA/MRI, diagnostic injection, and direct visualization with arthroscopy. ${ }^{18}$ Because of this, there is variability in the clinical indications for hip instability surgery. Moreover, there is a small percentage of patients that undergo revision hip arthroscopy for capsular instability who do not significantly improve. Given the current diagnostic methods, it can be quite difficult to identify these patients before revision surgery. We believe that standardized, quantitative approaches applied to the problem of post-operative hip instability may help increase the precision of indications and, that the measurements proposed in this study may provide a framework for future studies on the subject.

\section{Individual Measurement Utility}

The overall increase in effective intracapsular volume observed in the study cohort is related to the increase in volume in the anterosuperior proximal neck region where the interportal capsulotomy is most commonly created. The proximal anterosuperior hip capsular tissue in this patient population tends to be thinned, attenuated, or frankly absent. Although effective intracapsular volume was found to be significantly increased in the unstable postarthroscopy state, it is a global measure of capsular volume and does not independently measure the specific area of capsular deficiency. Thus, this measurement does not only identify iliofemoral ligament lesions but also may capture global capsular laxity or redundancy and may be influenced by cam resection volume. Key points in interpreting this measurement are understanding it in the context of thinned patulous capsular morphology, gross capsular deficiency with surrounding muscle fascia acting to contain the contrast, and iatrogenic communication between the hip capsule and the psoas sheath, all of which potentially contribute to instability. These MRA findings all influence measured effective capsular volume and may affect hip stability to different degrees.

The capsular retraction distance was found to be highly significant and is a useful measurement, particularly in the situation of a gross capsular rent. It is less useful, however, in redundant hip capsules when the healed tissue is continuous but thinned and elongated, as capsular retraction and leaflet edges may be difficult to discern or absent. Leaflet edges may be atrophied or may demonstrate thin fibrous tissue (pseudocapsule) that connects the two leaflets, confounding this measurement and likely contributing to its limited reliability. However, capsular retraction is a useful MRA finding when clearly identifiable.

The maximal anterior fluid depth measurement is sensitive to both capsular attenuation and gross capsular extravasation as both tend to create a pocket of fluid anterior to the femoral head-neck junction. The major limitation to this measurement is that it is 1 -dimensional. The maximal anterior fluid depth measurement does not assess the width of the capsular defect in the axis parallel to the femoral neck. Therefore it does not assess the overall size of the deficiency or region of missing ligament; however, it does provide a reliable estimation of anterosuperior capsular integrity. Future studies in this area should include correlation of hip/capsular biomechanics with MRA capsule morphologic parameters, as well as MRA correlation with hip functional outcomes.

\section{Limitations}

This study has several limitations. Regarding the comparator cohort for the instability group, the preoperative state was used. Because of this it is difficult to identify what proportion of the capsular changes can be attributable to postoperative healing and what proportion is true morphological change related to instability. A superior comparator cohort would be a group of patients without symptoms of instability after hip arthroscopy. However, the invasive nature of MRA limits the feasibility of such a study. In addition, previous MRI studies have demonstrated that the iliofemoral ligament is intact and slightly thickened postoperatively. ${ }^{8,9}$ Such findings are grossly different from those in our study, strengthening the argument that the capsular deficiencies observed here may be indicative of instability phenotype. In addition, increased capsular laxity and increased volume on arthrogram has been shown to be related to hip instability, but it has not previously been quantified. It should be noted, however, that further biomechanical and patient outcome data will be needed to better 
translate the findings in this manuscript to clinical decision making.

In addition, there are differences amongst how the hip capsule was initially managed at the index hip arthroscopy procedure in each case, which are attributable to the evolving technique of the primary author as well as some index surgeries being done outside of the study medical center. Thus, it is impossible to assess the durability of index repair techniques using this study design. However, the technique used for the index repair is less relevant for the central question of the present study, because all patients had clinical history and physical examination findings consistent with iatrogenic hip instability.

Intracapsular volume measurements are not formally defined in the literature. Most prior studies use saline solution or similar fluid injection in cadavers to estimate intracapsular volume. ${ }^{30-32}$ In the present study, nonnormal capsule geometry makes a definition of "intracapsular volume" even more difficult to define. However, with the defined measurement protocol described herein, the authors achieved high reliability of the measurement; however, it should be understood that capsular volume is a measure influenced by multiple factors and is likely less specific than, for example, capsule leaflet retraction, which implies a profound capsule structural defect. In addition, capsular volume could be affected by the cam resection, as a larger resection effectively increases capsular volume because of subtraction of bone from the femoral head-neck junction.

In addition, the MRA contrast volume injection was not completely standardized. MRAs obtained at the index arthroscopy at other institutions may have had different contrast injection protocols, and the volume of injectate used is somewhat user dependent. Despite this, consistent increases of fluid volume in the anterosuperior neck area and contrast extravasation and capsular defects in this region correlate with the increase in overall volume.

\section{Conclusion}

In comparison to the preoperative state, iatrogenic hip instability is associated with MRA findings that include increases in effective intracapsular volume, proximal anterosuperior and anteroinferior intracapsular cross sectional areas, maximum proximal anterosuperior fluid depth, and capsule retraction distance.

\section{References}

1. Bonazza NA, Homcha B, Liu G, Leslie DL, Dhawan A. Surgical trends in arthroscopic hip surgery using a large national database. Arthroscopy 2018;34:1825-1830.

2. Mei-Dan O, McConkey MO, Brick M. Catastrophic failure of hip arthroscopy due to iatrogenic instability: Can partial division of the ligamentum teres and iliofemoral ligament cause subluxation? Arthroscopy 2012;28:440-445.

3. Matsuda DK. Acute iatrogenic dislocation following hip impingement arthroscopic surgery. Arthroscopy 2009;25: 400-404.

4. Philippon MJ, Schenker ML, Briggs KK, Kuppersmith DA, Maxwell RB, Stubbs AJ. Revision hip arthroscopy. Am J Sports Med 2007;35:1918-1921.

5. Wylie JD, Beckmann JT, Aoki SK. Dislocation after hip arthroscopy for cam-type femoroacetabular impingement leading to progressive arthritis. JBJS Case Connect 20 15; 5:e80.

6. Bolia IK, Fagotti L, Briggs KK, Philippon MJ. Midterm outcomes following repair of capsulotomy versus nonrepair in patients undergoing hip arthroscopy for femoroacetabular impingement with labral repair. Arthroscopy 2019;35:1828-1834.

7. McCormick F, Slikker W 3rd, Harris JD, et al. Evidence of capsular defect following hip arthroscopy. Knee Surg Sports Traumatol Arthrosc 2014;22:902-905.

8. Weber AE, Kuhns BD, Cvetanovich GL, et al. Does the hip capsule remain closed after hip arthroscopy with routine capsular closure for femoroacetabular impingement? A magnetic resonance imaging analysis in symptomatic postoperative patients. Arthroscopy 2017;33:108-115.

9. Strickland CD, Kraeutler MJ, Brick MJ, et al. MRI evaluation of repaired versus unrepaired interportal capsulotomy in simultaneous bilateral hip arthroscopy: A double-blind, randomized controlled trial. J Bone Joint Surg Am 2018;100:91-98.

10. Guanche CA. Hip instability In: Operative Hip Arthroscopy. New York: Springer, 2012;339-348.

11. Wylie JD, Beckmann JT, Maak TG, Aoki SK. Arthroscopic capsular repair for symptomatic hip instability after previous hip arthroscopic surgery. Am J Sports Med 2016;44: 39-45.

12. Magerkurth O, Jacobson JA, Morag Y, Caoili E, Fessell D, Sekiya JK. Capsular laxity of the hip: Findings at magnetic resonance arthrography. Arthroscopy 2013;29:1615-1622.

13. Ng KCG, El Daou H, Bankes MJK, Rodriguez y Baena F, Jeffers JRT. Hip joint torsional loading before and after cam femoroacetabular impingement surgery. Am J Sports Med 2019;47:420-430.

14. Kurz AZ, Memon M, Williams D, Ayeni OR. Anterior capsule reconstruction of the native hip: A technique guide. Arthrosc Tech 2019;8:e1247-e1253.

15. Trindade CAC, Sawyer GA, Fukui K, Briggs KK, Philippon MJ. Arthroscopic capsule reconstruction in the hip using iliotibial band allograft. Arthrose Tech 2015;4: e71-e74.

16. Bolia I, Chahla J, Locks R, Briggs K, Philippon MJ. Microinstability of the hip: A previously unrecognized pathology. Muscles Ligaments Tendons J 2016;6:354-360.

17. Dangin A, Tardy N, Wettstein M, May O, Bonin N. Microinstability of the hip: A review. Orthop Traumatol Surg Res 2016;102:S301-S309.

18. O’Neill DC, Mortensen AJ, Cannamela PC, Aoki SK. Clinical and radiographic presentation of capsular iatrogenic hip instability after previous hip arthroscopy. Am J Sports Med 2020;48:2927-2932.

19. Arrieta C, Urrutia J, Besa P, et al. Automatic quantification of fat infiltration in paraspinal muscles using T2- 
weighted images: An OsiriX application. Biomed Signal Process Control 2020;57:101793.

20. Vellone V, Costantini AM, Ramieri V, Alunni Fegatelli D, Galluccio G, Cascone P. Unilateral condylar hyperplasia: A comparison with two open-source softwares. J Craniofac Surg 2020;31:475-479.

21. Poilliot A, Tannock M, Zhang M, Zwirner J, Hammer N. Quantification of fat in the posterior sacroiliac joint region applying a semi-automated segmentation method. Comput Methods Programs Biomed 2020;191:105386.

22. Cicchetti DV. Guidelines, criteria, and rules of thumb for evaluating normed and standardized assessment instruments in psychology. Psychological Assessment 1994;6: 284-290.

23. Byrd JWT, Jones KS. Diagnostic accuracy of clinical assessment, magnetic resonance imaging, magnetic resonance arthrography, and intra-articular injection in hip arthroscopy patients. Am J Sports Med 2004;32:1668-1674.

24. Packer JD, Foster MJ, Riley GM, et al. Capsular thinning on magnetic resonance arthrography is associated with intra-operative hip joint laxity in women. J Hip Preserv Surg 2020;7:298-304.

25. Domb BG, Stake CE, Lindner D, El-Bitar Y, Jackson TJ. Revision hip preservation surgery with hip arthroscopy: Clinical outcomes. Arthroscopy 2014;30:581-587.

26. Mortensen AJ, Metz AK, Froerer DL, Aoki SK. Hip capsular deficiency-a cause of post-surgical instability in the revision setting following hip arthroscopy for femoroacetabular impingement [published online November 17, 2021]. Curr Rev Musculoskelet Med. doi: 10.1007/ s12178-021-09732-5.

27. Woodward RM, Philippon MJ. Persistent or recurrent symptoms after arthroscopic surgery for femoroacetabular impingement: A review of imaging findings. J Med Imaging Radiat Oncol 2019;63:15-24.

28. Yeung M, Memon M, Simunovic N, Belzile E, Philippon MJ, Ayeni OR. Gross instability after hip arthroscopy: An analysis of case reports evaluating surgical and patient factors. Arthroscopy 2016;32: 1 196-1204.el.

29. Shin JJ, de SA DL, Burnham JM, Mauro CS. Refractory pain following hip arthroscopy: Evaluation and management. J Hip Preserv Surg 2018;5:3-14.

30. Waterman BR, Chen A, Neal WH, et al. Intra-articular volume reduction with arthroscopic plication for capsular laxity of the hip: A cadaveric comparison of two surgical techniques. Arthroscopy 2019;35:471-477.

31. Yen C-H, Leung H-B, Tse PY-T. Effects of hip joint position and intra-capsular volume on hip joint intra-capsular pressure: A human cadaveric model. J Orthop Surg Res 2009;4:8.

32. Schwarz N, Leixnering M, Hopf R, Jantsch S. Pressurevolume ratio in human cadaver hip joints. Arch Orthop Trauma Surg 1988;107:322-325. 